\title{
Etude Comparative des Techniques Chirurgicales dans la Chirurgie de la Cataracte au Niger
}

\author{
Abba Kaka H.Y. \\ Service d'Ophtalmologie Hôpital National de Niamey, Niger \\ Diori N.A. \\ Service d'Ophtalmologie Hôpital National Lamorde Niamey, Niger \\ Roufaye $L$. \\ Service d'Ophtalmologie Hôpital National de Niamey, Niger
}

Doi:10.19044/esj.2021.v17n17p180

Submitted: 12 April 2021

Accepted: 11 May 2021

Published: 31 May 2021
Copyright 2021 Author(s)

Under Creative Commons BY-NC-ND

4.0 OPEN ACCESS

Cite As:

Abba Kaka H.Y., Diori N.A. \& Roufaye L. (2021). Etude Comparative des Techniques Chirurgicales dans la Chirurgie de la Cataracte au Niger. European Scientific Journal, ESJ, 17(17), 180. https://doi.org/10.19044/esj.2021.v17n17p180

\section{Résumé}

Objectif: Le but de cette étude est de comparer les techniques chirurgicales dans la chirurgie de la cataracte au Niger. Patients et méthode: Il s'agissait d'une étude retrospective sur 12 mois du 1er Janvier au 31 Décembre 2012 portant sur les patients opérés de cataracte pendant les camps de chirurgie foraine dans les 8 regions du Niger. Résultats: Au total 1727 yeux cataractés ont été opérés dont 908 (52.58\%) par phaco-alternative et 819 (47.42\%) par l'extraction extra capsulaire. A J30 post opératoire la Phacoalternative a donné $29 \%$ de moyennes et bonnes acuités visuelles comparé à $27 \%$ pour l'extra-capsulaire. Les implants standards de 22 dioptries étaient les plus utilisés avec $26.21 \%$. Les implants de puissance entre 21 et 22 dioptries étaient responsables des meilleures acuités visuelles à J60. Conclusion: La Phaco-alternative comparée à l'extraction extra-capsulaire donnait de meilleurs résultats post-opératoires dans la chirurgie de la cataracte au Niger.

Mots clés : Chirurgie de la cataracte, technique chirurgicale, Niger 


\title{
Comparative Study in Cataract Surgical Technics in Niger
}

\author{
Abba Kaka H.Y. \\ Service d'Ophtalmologie Hôpital National de Niamey, Niger \\ Diori N.A. \\ Service d'Ophtalmologie Hôpital National Lamorde Niamey, Niger \\ Roufaye L. \\ Service d'Ophtalmologie Hôpital National de Niamey, Niger
}

\begin{abstract}
Aim: This paper focuses on comparing the surgical technics in cataract surgery in Niger. Patients and Method: It was a retrospective study which consisted of about 1,727 operated eyes of cataract during advance cataract surgical camps in the 8 regions of Niger. This happened over a period of 12 months from 1st January to 31st December of the year 2012. Résultats: A total of 1,727 cataract eyes were operated on during the study period. 908 patients $(52.58 \%)$ were operated by phaco-alternative and $819(47.42 \%)$ patients were operated by extra capsular extraction. On D30 postoperatively, phaco A gave $29 \%$ of good results compared to $27 \%$ for the extra-capsular. Standard 22 diopter implants were the most used in $26.21 \%$ of cases. Power of implants between 21 and 22 diopters were responsible for the best visual acuity at D60. Conclusion: Phaco-alternative technic compared to extra capsular gave better postoperative results in cataract surgery in Niger.
\end{abstract}

Keywords: Cataract surgery, surgical technic, Niger

\section{Introduction}

La cataracte première cause de cécité curable et constitue une des maladies cibles de l'initiative «Vision 2020, le droit à la Vue » qui vise à éliminer les causes de cécité évitable d'ici l'an 2020 (OMS/9 25février 2000). Le Niger fait partie des pays de l'Afrique de l'Ouest qui s'étaient fixés cet objectif.

Le traitement de la cataracte est purement chirurgical. Il consiste à l'ablation du noyau cristallinien opacifié (phacoexérèse) et la correction de l'aphaquie résiduelle. Deux techniques sont pratiquées actuellement au Niger notamment : l'extraction extra capsulaire classique (EECC) et la phaco alternative manuelle sans suture (PKA). D'autres techniques plus récentes notamment la Phacoemulcification(Phaco E ) et le Fentolaser sont pratiqués dans les pays développés. L'utilisation de ces dernières techniques sont limités dans nos pays en voie de développement à cause du cout élevé des appareils et du consommable mais aussi à cause de l'apprentissage plus difficile. La 
Phacoalternative est une méthode non motorisé et moins onéreuse alternative à la Phaco E. Ces techniques s'accompagnent de la pose d'un implant en chambre antérieure ou postérieure (Nadio, 2017). Une bonne prise en charge de la cataracte nécessiterait une chirurgie de qualité et un suivi post opératoire doué d'efficacité et d'efficience.

Le but de cette étude est ressortir les résultats comparatifs des 2 techniques chirurgicales utilisées en chirurgie de la cataracte au Niger.

\section{Patients et Méthode}

Il s'agissait d'une étude rétrospective portant sur les dossiers des patients opérés de la cataracte pendant les camps de chirurgie foraine du 1er Janvier au 31 Décembre de l'année 2012. Etaient inclus dans l'étude tous les dossiers des patients opérés de cataracte ayant des renseignements complets sur l'acuité visuelle, la puissance de l'implant et la technique opératoire. Nous avons étudié les paramètres suivants : la technique chirurgicale, l'acuité visuelle post-opératoire comparativement à la technique opératoire, l'acuité visuelle post-opératoire et la puissance de l'implant. Les patients se sont vus attribuer au hasard la technique d'EECC ou la technique de PKA manuelle. Dans le cas de l'EECC, le noyau de la cataracte a été extrait par une incision cornéo-limbique de $10 \mathrm{~mm}$, puis le cortex a été aspiré et le cristallin artificiel implanté dans la chambre postérieure. L'incision a été suturée par des points séparés au fil 10/0. Dans le cas de la PKA, un tunnel scléral a été construit en utilisant un kératome et le noyau cristallinien luxé dans la chambre antérieure. Le noyau a ensuite été extrait en utilisant une solution viscoélastique. L'aspiration du cortex et l'implantation du cristallin artificiel étaient semblables à la technique d'EECC, mais il n'était pas nécessaire de suturer car la plaie était auto étanche.

Ce caractère faiblement invasif autorise la réalisation d'une chirurgie ambulatoire. Le suivi des patients a été effectué à $\mathrm{J} 1, \mathrm{~J} 30$ et à $\mathrm{J} 60$ post opératoire.

Les résultats ont été classés en trois groupes : bon, moyen et mauvais selon les directives et recommandations de l'OMS concernant les résultats postopératoires de la chirurgie de la cataracte avec implant intraoculaire comme le montre le tableau ci-dessous

$\begin{array}{llll}\text { Résultat } & \text { AVL } & \text { AVSC } & \text { AVAC } \\ \text { Bon } & 10 / 10-3 / 10 & >80 \% & >90 \% \\ \text { Moyen } & <3 / 10-1 / 10 & <15 \% & <5 \% \\ \text { Mauvais } & <1 / 10 & <5 \% & <5 \%\end{array}$

$(\mathrm{AVL}=$ acuité Visuelle de Loin, $\mathrm{AVSC}=$ acuité visuelle sans correction, $\mathrm{AVAC}=$ acuité visuelle avec correction) 
Aucun des patients n'a bénéficié d'un calcul de la puissance de l'implant et aucun n'a eu une correction optique en post-opératoire.

\section{Résultats}

Le Tableau I ressort les deux techniques opératoires utilisés avec précision de l'utilisation d'un implant intra-oculaire ou pas

Tableau I. Répartition des opérés selon la technique chirurgicale et l'implantation

\begin{tabular}{|l|l|l|}
\hline Type chirurgie & Nombre & Pourcentage \\
\hline EEC/ICA & 16 & $0.93 \%$ \\
EEC/ICP & 783 & $45.33 \%$ \\
EEC/SIMPLE & 20 & $1.16 \%$ \\
PHACOA/ICA & 08 & $0.47 \%$ \\
PHACOA/ICP & $\mathbf{8 9 8}$ & $\mathbf{5 1 . 9 9 \%}$ \\
PHACOA/SIMPLE & 02 & $0.12 \%$ \\
\hline Total & 1,727 & $100 \%$ \\
\hline
\end{tabular}

(EEC/ICA = extraction extra capsulaire/implant de chambre antérieure $\mathrm{EEC} / \mathrm{ICP}=$ extraction extra capsulaire/ implant de chambre postérieure PHACO A/ICA= phacoalternative/ implant de chambre antérieure PHACO A/ICP = phacoalternative/ implant de chambre postérieure)

Au total 908 patients soit $52.58 \%$ ont été opérés par la méthode phacoalternative avec ou sans implant. 
Tableau II. comparaison entre acuité visuelle post opératoire sans correction et technique opératoire à J1, J30 et J60

\begin{tabular}{|l|l|l|l|l|}
\hline \multirow{2}{*}{$\begin{array}{l}\text { Jour de control } \\
\text { Et }\end{array}$} & \multicolumn{2}{l|}{ Qualité de l'acuité visuelle } & \multirow{2}{l|}{ Perdus de vue } \\
\hline Technique opératoire & Mauvaise & Moyenne & Bonne & \multirow{2}{*}{ Nbr/\% } \\
\cline { 2 - 5 } & Nbr/\% & Nbr/\% & Nbr/ \% & \\
\hline $\begin{array}{l}\text { J1 } \\
\text { EEC / ICP }\end{array}$ & $355 / 20.55$ & $313 / 18.12$ & $106 / 6.13$ & $9 / 0.9 \%$ \\
\hline $\begin{array}{l}\text { J1 } \\
\text { PHACO A / ICP }\end{array}$ & $347 / 20.09$ & $401 / 23.21$ & $144 / 8.33$ & $6 / 0.34$ \\
\hline $\begin{array}{l}\text { J30 } \\
\text { EEC / ICP }\end{array}$ & $91 / 5.26$ & $217 / 12.50$ & $244 / 14.12$ & $229 / 13.25$ \\
\hline $\begin{array}{l}\text { J30 } \\
\text { PHACO A / ICP }\end{array}$ & $60 / 3.47$ & $216 / 12.50$ & $285 / 16.50$ & $337 / 19.51$ \\
\hline $\begin{array}{l}\text { J60 } \\
\text { EEC / ICP }\end{array}$ & $31 / 1.79$ & $144 / 8.33$ & $182 / 10.53$ & $426 / 24.66$ \\
\hline $\begin{array}{l}\text { J60 } \\
\text { PHACO A / ICP }\end{array}$ & $19 / 1.10$ & $128 / 7.41$ & $185 / 10.71$ & $566 / 32.77$ \\
\hline
\end{tabular}

A J30 post opératoire la phaco A a donné $29 \%$ de moyens et bons résultats comparé à $27 \%$ pour l'extra-capsulaire.

Tableau III. Répartition des opérés selon la puissance des implants utilisés en dioptries

\begin{tabular}{|l|l|l|}
\hline Puissance Implant & Fréquence & Pourcentage \% \\
\hline 18,5 & 5 & $0.3 \%$ \\
19 & 6 & $0.4 \%$ \\
19,5 & 54 & $3.2 \%$ \\
$\mathbf{2 0}$ & $\mathbf{2 4 4}$ & $\mathbf{1 4 . 1 3 \%}$ \\
20,5 & 202 & $12.1 \%$ \\
$\mathbf{2 1}$ & $\mathbf{3 8 9}$ & $\mathbf{2 2 . 8 1 \%}$ \\
21,5 & 285 & $17.1 \%$ \\
$\mathbf{2 2}$ & $\mathbf{4 4 7}$ & $\mathbf{2 6 . 2 1 \%}$ \\
22,5 & 24 & $1.4 \%$ \\
23 & 49 & $2.9 \%$ \\
\hline Total & 1,705 & $100 \%$ \\
\hline
\end{tabular}


Sur les 1,727 patients opérés, 1,705 avaient bénéficiés de la mise en place d'un implant intraoculaire, les implants de 22 dioptries étaient les plus utilisés avec $26.21 \%$.

Tableau IV. comparaison entre l'acuité visuelle post opératoire sans correction et la puissance des implants

\begin{tabular}{|l|l|l|l|l|}
\hline $\begin{array}{l}\text { Acuité visuelle à J60 } \\
\text { Puissance de l'implant }\end{array}$ & Mauvaise & Moyenne & Bonne & Perdus de vue \\
\hline 18,5 & 2 & 1 & 1 & 1 \\
\hline 19 & 0 & 1 & 1 & 4 \\
\hline 19,5 & 1 & 7 & 20 & 26 \\
\hline 20 & 8 & 51 & 53 & 120 \\
\hline 20,5 & 8 & 34 & 53 & 107 \\
\hline 21 & 14 & 57 & 71 & 235 \\
\hline 21,5 & 14 & 50 & 76 & 145 \\
\hline 22 & 6 & 70 & 78 & 281 \\
\hline 22,5 & 2 & 3 & 2 & 17 \\
\hline 23 & 0 & 4 & 2 & 43 \\
\hline Puissance non spécifiée & 2 & 4 & 10 & 42 \\
\hline
\end{tabular}

Les implants de puissance entre 21 et 22 dioptries sont responsables des meilleures acuités visuelles à J60.

\section{Discussion}

La cataracte est la première cause de cécité curable dans le monde et aussi au Niger ou elle constitue 45\% des causes identifiées de cécité (OMS/9 25février 2000). Ce taux élevé fait de cette pathologie un véritable problème de santé publique. Le cout onéreux et l'accessibilité difficile à la chirurgie de cataracte font d'avantage obstacle et augmentent le nombre de cas non opérés dans le pays. L'évolution des techniques opératoires de la cataracte ont fait que l'extraction intra-capsulaire a été abandonnée au profit de l'extraction extra capsulaire et plus récemment de la phacoalternative (PhacoA). Ces deux dernières techniques ont fait leur entrée dans la pratique chirurgicale de la cataracte. Dans cette étude que nous présentons 1,727 yeux avaient été opérés de cataracte. Deux techniques chirurgicales ont été pratiquées sur ces patients notamment l'extraction extra capsulaire dans $47.42 \%$ des cas et la phacoalternative sur $52.58 \%$ des yeux. Pour corriger l'aphaquie chirurgicale ainsi 
créee, un implant est mis en place pour remplacer le cristallin. La position idéale est dans le sac capsulaire en chambre postérieure. L'implant peut aussi être placé en chambre antérieure au cas où le support anatomique (la capsule postérieure) n'est plus en mesure de garder l'implant en place. Dans d'autres cas encore plus désespérants aucun implant n'est mis en place, alors le patient était appelé à avoir une implantation secondaire ou une correction optique post opératoire (verres d'aphaque). Au total 908 yeux soit $52.58 \%$ ont été opérés par la méthode Phaco-alternative avec ou sans implant. Ce chiffre est supérieur à celui rapporté par Diarra SM et al. au Mali qui est de 38\% (Diarra, 2020), de même que Guirou N et al. qui ont aussi retrouvé $47.36 \%$ de PhacoA (Guirou, 2013). Par contre 51\% des patients Sowagnon TYC opérés par PhacoA (Swagnon, 2014) ce chiffre se rapproche du notre. De ce paramètre nous en concluons que la phacoalternative rentre progressivement dans les habitudes des chirurgiens africains. L'étude de Ruit $S$ au Népal a conclu que la phacoA était la meilleure méthode pour éliminer la cataracte dans les pays en développement vu le cout moins onéreux et le temps opératoire court (Ruit, 1999). L'acuité visuelle est un indice d'évaluation de la qualité de la chirurgie de la cataracte. Comment énuméré dans la méthodologie de cette étude elle a été classée en bonne, moyenne et mauvaise selon les recommandations de l'OMS concernant les résultats postopératoires de la chirurgie de la cataracte avec implant intraoculaire. En comparant les deux techniques (Tableau II), l'acuité post opératoire dans la phaco $\mathrm{A}$ a donné de meilleurs résultats. Ce concept est accepté par tous les auteurs pré-cités et concorde avec les données de la littérature (Oye, 2006; Schemann, 2006). Le faible taux d'astigmatisme cornéen, la petite incision, le faible taux de complications per et postopératoires sont entre autres les facteurs en faveur de la PhacoA, mais la présentation tardive dans nos régions limite les bénéfices liés à cette technique. Les implants standards étaient utilisés par manque de biométrie et ceux de 22 dioptries étaient les plus utilisés tout comme dans l'étude de Diarra SM. Pour Guirou N l'implant calculé était indisponible dans seulement $1 \%$ des cas, et 95\% des patients de Sowagnon TYC n'ont pu bénéficier d'une échobiometrie. Par contre tous les patients de Assoumou PA et al. avaient bénéficié d'une biométrie oculaire en mode contact en pré-opératoire avec calcul de la puissance de l'implant intraoculaire emmétropisant selon la formule SRK/T (Assoumou, 2018). L'étude comparative à J60 entre l'acuité visuelle et la puissance de l'implant a fait ressortir dans notre étude que les implants de puissance 22 dioptries avaient plus de bons résultats, suivis des 21 dioptries. D'une manière générale nos résultats sur l'acuité visuelle restent inférieurs aux normes de l'OMS et à ceux des autres études, le grand nombre d'absents (59.11\% à J60) en visite retour tout comme le manque de biométrie et de correction optique post-opératoire chez ces patients semblent être des facteurs incriminants. 


\section{Conclusion}

La chirurgie de la cataracte reste un défit dans les pays sous développés ou le taux de cécité imputable à celle-ci dépasse $40 \%$. Cette demande élevée appelle à des techniques accessibles comme la Phacoalternative. L'acuité visuelle étant l'indicateur le plus important dans la réussite de la chirurgie de la cataracte, cette série nous montre des meilleurs résultats chez les patients ayant étés opérés par la méthode phaco-alternative. Cette technique étant d'apprentissage moins difficile, ayant un cout moindre et un temps opératoire plus court doit être la technique de référence pour les pays à faible revenus dans leurs perspectives d'atteindre les objectifs de la vision 2020.

Conflits d'intérêts: aucun

Contribution des auteurs: tous les auteurs ont participé au recensement à l'élaboration et à la rédaction de cet article.

Remerciements: Au personnel du service d'Ophtalmologie de l'Hôpital National de Niamey.

\section{References:}

1. Assoumou, PA., Mba Aki, T., Dohvoma, A et al.(2018). La Phacoalternative Manuelle sans Suture dans la chirurgie de masse de la cataracte de l'adulte à Libreville, Health Sci. Dis:19 (4)1.

2. Diarra, SM., Guindo, A., Saye, G et al. (2020). Résultats fonctionnels de la chirurgie de la cataracte de l'adulte dans le district sanitaire de la commune VI à Bamako (Mali) : bilan de deux campagnes de chirurgie de masse. Jaccr Africa; 4(1): 314-319.

3. Guirou, N., Napo, A., Dougnon, A et al. (2013). Résultats fonctionnels de la chirurgie de la cataracte de l'adulte; Journal français d'ophtalmologie, 36, 19 - 22.

4. Initiative mondiale pour l'élimination de la cécité évitable Lancement de l'Initiative Vision 2020 en Afrique Francophone. Communiqué de presse OMS/9 25février 2000.

5. Nadio, T., Napo, A., \& Balde, R. (2017). Extraction extra capsulaire versus Phacomanuelle sans sutures dans le traitement de la cataracte de l'adulte Au Chu-Iota. Mali Medical, Tome Xxxii N³,p 16 - 19.

6. Oye, JE., Kuper, H., Dineen, B., Befidi-Mengue, R., \& Foster, A. (2006). Prevalence and causes of blindness and visual impairment in Muyuka: a rural health district in South West Province, Cameroon. Br J Ophthalmol;90:538-42.

7. Ruit, S., Tabin, GC., Nissman, SA., Paudyal, G., \& Gurung, R. (1999). Low-cost high-volume extra capsular cataract extraction 
with posterior chamber intraocular lens implantation in Nepal; Ophtalmol; 106(10):1887-92.

8. Sowagnon, TYC., Kouassi, FX., Koné, S et al. (2014) Etude comparative de l'extraction extracapsulaire (EEC) manuelle versus chirurgie de la cataracte à petite incision. manuelle: expérience du centre Ophtalmologique protestant, Méthodiste Bartimée de Dabou, Revue SOAO N ${ }^{\circ}$ 02, 33-37.

9. Schemann, JF., Inocencio, F., de Lourdes Monteiro, M et al. (2006). Blindness and low vision in Cape Verde Islands: results of a national eye survey. Ophthalmic Epidemiol;13:219—26.

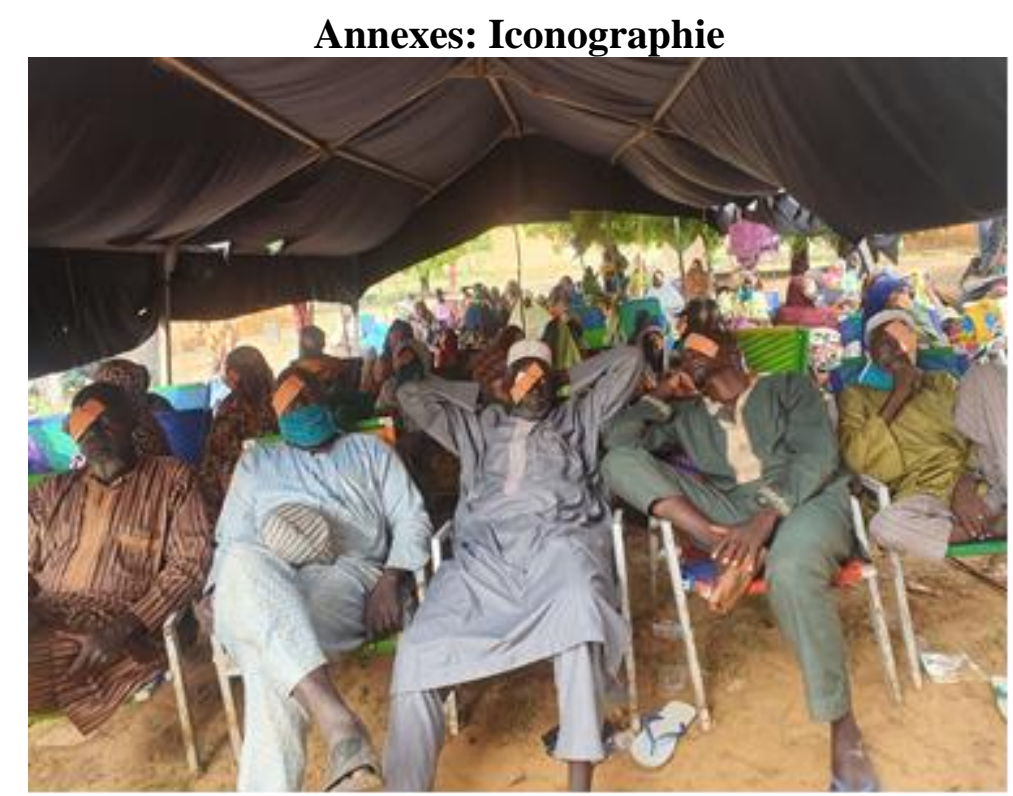

Photo 1- Patients a J1 post-opératoire (source: programme de Santé Oculaire du Niger)

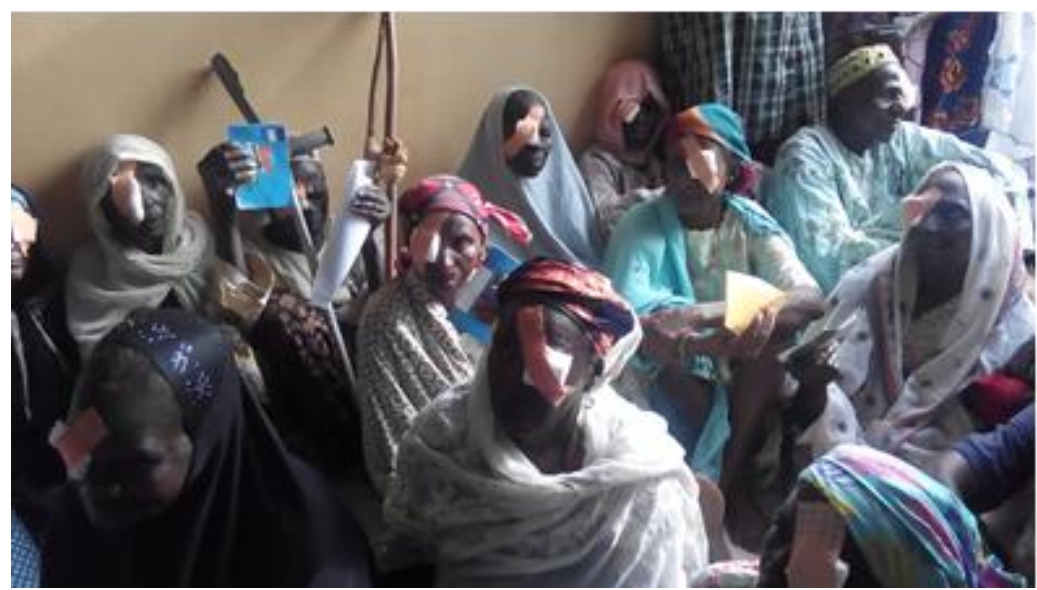

Photo 2- Patientes à J1 post-opératoire (source: programme de Santé Oculaire du Niger) 